Ottmar Ette

(Potsdam)

\title{
VOM AUFTAUCHEN AMERIKAS ZUM VERSCHWINDEN EUROPAS. AMERIKANISCHE TRÄUME VON KOLUMBUS BIS BAUDRILLARD
}

I.

Am Beginn der heutigen Gestalt Amerikas steht eine Vogelschau. In dem uns von Bartolomé de Las Casas überlieferten Bordbuch des Cristóbal Colón finden wir, datiert auf den Tag des Herrn, Sonntag, den 7. Oktober 1492, den folgenden Eintrag:

Como en la tarde no viesen tierra, la que pensaban los de la carabela Niña que habían visto, y porque pasaban gran multitud de aves de la parte del norte al Sudueste, por lo cual era de creer que se iban a dormir a tierra, o huían quizá del invierno, que en las tierras de donde venían debía que querer venir, por esto el Almirante acordó dejar el camino del Oueste, y pone la proa hacia Ouesu(du)este con determinación de andar dos días por aquella vía. Esto comenzó antes una hora del sol puesto.

Die leichte Kursänderung, die Kolumbus an jenem denkwürdigen Tag ausführen ließ, und welche die Schiffe, die zuvor nach Westen gesegelt waren, nun in eine südwestliche Fahrtrichtung brachten, hatte weitreichende Folgen für die sogenannte Entdeckung Amerikas, die Geschichte ihrer Eroberung und Kolonisation wie auch die geokulturelle und letztlich geopolitische Konfiguration der amerikanischen Hemisphäre. Denn hätte Kolumbus den Kurs beibehalten und eine drohende Meuterei auf der dann längeren Fahrtstrecke überstanden, er wäre wohl mit Hilfe des starken Golfstroms an jenem Teile Amerikas gelandet, der uns heute als der Sunshine State bekannt ist: an der Küste Floridas. Schon Washington Irving war auf diese Stelle des Bordbuches gestoßen und hatte in seiner berühmten Biographie des Admirals auf die möglichen Folgen einer Fortsetzung der Fahrt nach Westen aufmerksam gemacht; Alexander von Humboldt, der wohl beste Kenner der damals zugänglichen Literatur zur Entdeckungs- und Erobe-

1 Cristóbal Colón (1985: 86-87); Verweise auf diese Textausgabe werden im Weiteren unter Verwendung der Sigle DAB sowie der entsprechenden Seite im fortlaufenden Text angegeben. 
rungsgeschichte Amerikas, hatte Irvings Überlegungen zur Kenntnis genommen und seinerseits auf diesen $»$ Umstand von unermesslicher Wichtigkeit« hingewiesen, »da er den Vereinigten Staaten statt einer protestantischen englischen Bevölkerung eine katholische spanische hätte geben können $\ll^{2}$.

Was war an Bord der spanischen Schiffe vor sich gegangen? Bereits am 6. Oktober, zu einem Zeitpunkt, als die von Kolumbus eigenhändig vorgenommenen Fälschungen der Angaben über zurückgelegte Distanzen nichts mehr fruchteten und die erschöpften Mannschaften der drei Karavellen an Widerstand gegen eine Fortsetzung der Fahrt nach Westen dachten, hatte Martín Alonso Pinzón, der als erfahrener Seemann bei den Matrosen nicht nur seines Schiffes in höherem Ansehen stand, vorgeschlagen, die Fahrtrichtung nach Südwesten abzuändern. Doch der Transkription des Bordbuches entnehmen wir an jenem Tag: »Al Almirante pareció que no« (DAB, 86). ${ }^{3}$ Erst am folgenden Tag willigte Kolumbus, wie wir sahen, unter Hinweis auf den Flug der Vogelschwärme in eine Änderung der Fahrtrichtung ein. Er griff dabei, wie Humboldt anmerkte, auf die Erfahrung der Portugiesen zurück, "welche den größeren Theil der Inseln, die sie besitzen«, aufgrund ihrer »Beobachtung des Fluges der Vögel entdeckt« hätten (KU, Bd. 1, 213). Die Seeleute willigten zunächst ein, zumal man auch während der folgenden Tage Zeichen nahen Landes wahrnahm; doch nachdem sie noch während der ganzen Nacht Vögel hatten vorbeifliegen hören, begehrten sie am 10. Oktober gegen Kolumbus auf, der sie ein letztes Mal mit Versprechungen beruhigen konnte. Am folgenden Tag, dem 11. Oktober, aber sah ein Matrose namens Juan Rodríguez Bermejo, der unter dem Namen Rodrigo de Triana in Geschichte und Legendenbildung Eingang fand (DAB, 88-89), das ersehnte Land, eine Meldung, die sich im Gegensatz zu jener der Niña vom 7. Oktober, am folgenden Tage bestätigte. Es waren die Vögel gewesen, welche die drei spanischen Schiffe in die Inselwelt der Karibik geführt und damit den weiteren Fortgang der Weltgeschichte nicht unwesentlich mitbeeinflusst hatten. Nicht zu Unrecht kommentierte Alexan-

2 Alexander von Humboldt (1836: Bd. 2, 111); Verweise auf diese Textausgabe finden sich im Weiteren unter Verwendung der Sigle KU sowie der entsprechenden Seite im fortlaufenden Text.

3 Die von Colón angeführten Gründe für die Ablehnung sind wenig überzeugend, so dass Luis Arranz in seinem Kommentar zu dieser Passage des Bordbuchs auch zu der Einschätzung gelangte, dass Kolumbus lediglich dem Eindruck entgegenwirken wollte, er habe diesen Entschluss auf Veranlassung Pinzóns getroffen (DAB, 87, n. 36). 
der von Humboldt in seinem überwiegend der Figur des Kolumbus gewidmeten und ursprünglich in französischer Sprache erschienenen Examen critique:

Niemals hat der Flug eines Vogels gewichtigere Folgen gehabt; Denn die Aenderung des Windstriches am 7. Oktober entschied die Richtung, nach welcher die ersten Ansiedelungen der Spanier in Amerika Statt finden sollten und gefunden haben. (KU, Bd. 2, 114-115)

Es mag vielleicht kein Zufall sein, dass dieses Forschungskolloquium zu Ehren von Dieter Janik unter dem Thema »Selbstvergewisserung am Anderen oder Der fremde Blick auf das Eigene« just an einem Freitag inauguriert wurde. Denn es war an einem Freitag, dem 3. August 1492, dass Kolumbus die Segel gesetzt hatte (»Partimos viernes 3 días de agosto de 1492 años, de la barra de Saltes, a las ocho horas « [DAB, 73].), und an einem Freitag war es, dass Kolumbus erstmals Fuß auf amerikanischen Boden setzte und die Zeichen spanischer Macht entfaltete:

Sacó el Almirante la bandera real, y los capitanes con dos banderas de la cruz verde, que llevaba el Almirante en todos los navíos por seña, con una $\mathrm{F}$ y una $\mathrm{Y}$, encima de cada letra su corona. (DAB, 90$)^{5}$

Damit beginnt nun die Deutung jener sich dem Blick des Kolumbus darbietenden Zeichen, eine Lektüre, die schon bei Humboldt, vor allem aber dann im Gefolge strukturalistischer und poststrukturalistischer Semiotik und Semiologie ein um das andere Mal ihrerseits gedeutet wurde. ${ }^{6}$ Vergessen wir darüber aber nicht die Tatsache, dass es die Deutung eines Vogelfluges und damit ein Augur - gleichviel, ob es sich dabei um Kolumbus oder Pinzón handelte - war, der als Interpret der Zeichen am Himmel in gewisser Weise

4 Zur Entwicklung des Kolumbus-Bildes in den Schriften Alexander von Humboldts cf. Ottmar Ette (1992). Was, so bliebe noch zu klären, hatte Pinzón auf den Gedanken einer Kurskorrektur gebracht? Humboldt ging auch dieser Frage in seinen Kritischen Untersuchungen (1836: Bd. 2, 114) nach: "Vallejo, ein aus Moguer gebürtiger Seemann, erzählt ganz naiv in den Proceßverhandlungen, daß >Pinzon am Abend habe Papageien vorüberfliegen sehen und gewußt habe, daß diese $V o ̈-$ gel nicht ohne besonderen Grund nach Süden hinflögen . « Dass es sich in den Worten des Seemanns um Papageien handelte, dürfte Humboldt, der Papageien viel abzugewinnen wusste, zusätzlich fasziniert haben; cf. auch den nicht ganz ernsten Aufsatz des Verfassers (1988).

5 Wir wollen dabei vergessen, dass der Freitag - worauf bereits Humboldt in Rückgriff auf frühe Kritiker des Kolumbus und der Entdeckungsgeschichte hinwies - »in der ganzen Christenheit als ein Tag von übler Vorbedeutung für den Beginn einer Unternehmung angesehen wurde« (KU, Bd. 2, 112).

6 Am erfolgreichsten war sicherlich der Band von Tzvetan Todorov (1985 [1982]). 
die Konzentration der Spanier auf den Süden und damit die vorübergehende Schaffung eines $>$ Leerraumes $<$ im Norden vorgab.

Die sich anschließende Fahrt des künftigen Admirals der Katholischen Könige durch die amerikanische Inselwelt, die noch ihrer europäischen Namen und ihren Mehrfachbenennungen als Antillen, als Karibik, als Westindien usw. harrte, wurde zur hermeneutischen Bewegung eines Zeichenlesers, der nicht nur seine Lesarten, sondern selbst seine Zeichen aus der Alten Welt mitgenommen hatte und nun auf die Küstensäume einer ihm noch unbekannten und doch so vertrauten Welt projizierte. So findet sich, um nur ein Beispiel anzuführen, unter dem 15. Januar 1493 in Las Casas' Transkription die folgende Eintragung:

Dice que se quiere partir porque ya no aprovecha nada detenerse, por haber pasado aquellos desconciertos (debe decir del escándalo de los indios). Dice también que hoy ha salido que toda la fuerza del oro estaba en la comarca de la Villa de la Navidad de Sus Altezas, y que en la isla de Carib había mucho alambre y en Matinino, puesto que será dificultoso en Carib, porque aquella gente diz que come carne humana, y que de allí se parecía la isla de ellos, y que tenía determinado de ir a ella, pues está en el camino, y a la de Matinino, que diz que era poblada toda de mujeres sin hombres, y ver la una y la otra, y tomar diz que algunos de ellos. (DAB, 191)

Diese Passage zeigt deutlich, in welcher Weise sich bereits im Bordbuch der ersten Reise Bruchstücke geographischer Vorstellungen und Illusionen, etymologischer Fehldeutungen, aus der griechischen Antike stammender Mythen oder ganz materiell orientierter Wunschträume zu einem bricolage formieren, in welchem Goldgier, Amazonentrauma, Anthropophagieangst und Benennungslust als Stationen und Elemente eines Weges erscheinen, den Kolumbus nicht aus den Augen verliert, führt er ihn doch zurück nach Spanien, zur Verkündigung des von ihm Gesehenen - und zum Entwurf jener amerikanischen Träume, die sich von Beginn an als komplexe Verschachtelung und Vergleichzeitigung abendländischer Kulturfragmente zu erkennen geben. Erst die Rückkehr nach Europa - und die sich anschließenden frühen Berichte von jener Entdeckung - machen es möglich, dass sich fortan ein Gutteil der Neuen in der Alten Welt bildet. ${ }^{7}$ Die amerikanischen Träume aber werden zwanghaft und zwangsweise in amerikanische Wirklichkeiten verwandelt. 
Der berühmte Aphorismus des Georg Christoph Lichtenberg, demzufolge der Tag, an dem der Indianer Kolumbus entdeckt habe, ein schlechter Tag für den Indianer gewesen sei, erwies sich bald nicht mehr nur in der künftig als Karibik bezeichneten Region als eine grausame Wahrheit, die durch die Umkehrung der Entdeckungsmetaphorik zum Vorschein gebracht wurde. Der sich an den Indianern vollziehende Genozid erfasste rasch auch die Bewohner des Festlands - nicht jener Insel Kuba, die Kolumbus für Festland hielt und seinen Männern den Schwur auf diesen Glauben abverlangte, sondern jener Küsten, die ein gewisser Amerigo Vespucci als Küstensäume eines Kontinents, einer Neuen Welt, erkannte. Bereits im Jahre 1500 - als weiter südlich aufgrund eines Sturms die nach Osten ragende Spitze des heutigen Brasilien von den Portugiesen eher zufällig >entdeckt und in Besitz genommen wird - nehmen die amerikanischen Träume kartographische Gestalt an in jener Karte, die Juan de la Cosa, der Kolumbus auf dessen zweiter Reise begleitet und zusammen mit Vespucci auch an der Expedition Alonso de Ojedas 1499 teilgenommen hatte, entwarf. ${ }^{8}$ Hier entstehen die Umrisse und Konturen einer Weltregion, in deren Zentrum sich eine Inselwelt befindet, deren Erkundung schon weiter fortgeschritten ist, und die den südlichen mit dem nördlichen Teil des Kontinents verbindet. Dabei ist auf der Karte von Juan de la Cosa der Norden des karibischen Beckens, des amerikanischen Mittelmeers, als Besitztum der Katholischen Könige markiert und festgehalten.

Noch kann man jenen Bereich erst erahnen, auf den sich dann zunächst die oftmals spontane, ungesetzliche, sich bisweilen explosionsartig vollziehende Expansion der Spanier konzentrieren wird. Doch Erkundungsfahrten von Francisco Hernández de Córdoba und Juan de Grijalva in den Jahren 1517 und 1518 bringen größere Klarheit über den Küstenverlauf und zugleich die Spanier erstmals in Kontakt mit einer wohlorganisierten und ausdifferenzierten indigenen Gesellschaft. Kurze Zeit später vollzieht sich die Eroberung jenes Reiches von Anáhuac mit seinem Zentrum Tenochtitlán, das die Männer um Hernán Cortés zwischen 1519 und 1521 unter Ausnutzung der Gegensätze und Feindseligkeiten zwischen den verschiedenen indianischen Völkern in ihre Gewalt bringen und unter dem Na-

8 In seinen Kritischen Untersuchungen (1836: Bd. 1, 16-17) machte Humboldt darauf aufmerksam, dass er zusammen mit Walkenaer 1832 »das Vergnügen« gehabt hatte, »den Urheber und das Datum« dieser Karte der Neuen Welt zu erkennen. 
men Nueva España dem fast nahtlos von der Reconquista in die Conquista umschwenkenden und umdenkenden Spanien einverleiben sollten. Der radikale Bruch mit Diego Velázquez, dem Gouverneur von Kuba, und die Gründung der Stadt Veracruz an der Karibikküste des heutigen Mexiko, das geographisch gesehen ja zu Nordamerika zählt, reichen nicht aus als Fundament der amerikanischen Träume des Cortés, die zugleich das Projekt einer Eroberung und das Projekt eines Staates und damit nicht nur militärische, sondern eminent politische Projektionen sind. Um seine Visionen in die Tat umzusetzen, muss er den Europäern, den spanischen Soldaten nicht nur die eigenen Träume vermitteln, sondern ihnen auch den Rückzug abschneiden. In seinem zweiten Brief an den Emperador Carlos $V$ stellt er am 30. Oktober 1520 trocken seine Vorgehensweise dar:

\begin{abstract}
Y porque demás de los que por ser criados y amigos de Diego Velázquez tenían voluntad de se salir de la tierra, había otros que por verla tan grande y de tanta gente y tal, y ver los pocos españoles que éramos, estaban del mismo propósito creyendo que si allí los navíos dejase, se me alzarían con ellos, y yéndose todos los que de esta voluntad estaban, yo quedaría casi solo, por donde se estorbara el gran servicio que a Dios y a vuestra alteza en esa tierra se ha hecho, tuve manera como, so color que los dichos navíos no estaban para navegar, los eché a la costa por donde todos perdieron la esperanza de salir de la tierra. Y yo hice mi camino más seguro y sin sospechas que vueltas las espaldas no había de faltarme la gente que yo en la Villa había de dejar. ${ }^{9}$
\end{abstract}

Auch Cortés ist sich seines Weges bewusst, der ihn ins Innere eines großen und bevölkerten Landes führt. Zugleich aber setzt er seine Männer fest, die ebenso wenig Gefallen wie jene des Kolumbus verspürt zu haben scheinen, dem ihnen vorgegebenen Weg zu folgen. Kolumbus hält gegen alle sich abzeichnende Meuterei seine Seeleute auf den Schiffen fest, während Cortés jedweden Aufstand sofort gewaltsam und geschickt unterbindet und seine Soldaten von den Schiffen abschneidet. Auch in den Cartas de relación zeichnet sich eine Welt ab, die sich als bricolage von Elementen verschiedenster (und nunmehr, nicht zuletzt dank der von Cortés >benutzten< Übersetzerin und lengua Malinche, auch autochthoner) Herkunft erweist. Wie bei Kolumbus werden auch in den unablässigen Feldzügen und Manövern des Cortés diese Elemente in beständiger Bewegung gehalten. Descubrimiento und Conquista sind als hermeneutische Bewegungsmuster einander

9 Hernán Cortés (1985: 84). 
wesensverwandt, ihre Bezugspunkte aber haben - die Zerstörung der Schiffe zeigt es geradezu emblematisch an - gewechselt. Von nun an wird der Binnenraum Amerikas zur Projektionsfläche für die amerikanischen Träume der Eroberer.

Im Verlauf des dritten Jahrzehnts des 16. Jahrhunderts versuchten mehrere spanische Expeditionen, die Ostseite Nordamerikas von der Küste des zunächst für eine Insel gehaltenen Florida bis hinauf zu den Küsten Labradors nach einer Durchfahrt zum Pazifik abzusuchen. Zugleich vergab Carlos $\mathrm{V}$ wiederholt die Erlaubnis zu Eroberung und Kolonisierung von Gebieten, die im heutigen Nordamerika liegen: Noch immer waren die nahe gelegenen Karibikinseln gleich riesigen Schiffen Drehscheiben für Informationen und Ausgangspunkte für Expeditionen. Pánfilo de Narváez, der zusammen mit Hernán Cortés unter Diego Velázquez maßgeblich an der Eroberung Kubas beteiligt gewesen war, und der im Auftrag des mit ihm befreundeten Gouverneurs von Kuba vergeblich den vertragsbrüchigen Hernán Cortés bei dessen Unterwerfung Anáhuacs hatte festnehmen wollen, erhielt vom Emperador das Recht zur Eroberung des 1512 entdeckten Florida. An seiner Expedition nimmt jener Mann teil, der mit seinen geradezu unglaublichen Erlebnissen, aber auch mit seinen Schriften in die Geschichte des amerikanischen Kontinents einging: Álvar Núñez Cabeza de Vaca. Seine Narrativa, die unter dem Titel Naufragios berühmt werden sollte, beginnt wie viele relaciones von Konquistadoren:

\begin{abstract}
A 17 días del mes de junio de 1527, partió del puerto de San Lúcar de Barrameda el gobernador Pánfilo de Narváez, con poder y mandado de Vuestra Majestad para conquistar y gobernar las provincias que están desde el Río de las Palmas hasta el cabo de la Florida, las cuales son en Tierra Firme; y la armada que llevaba eran cinco navíos, en los cuales poco más o menos, irían seiscientos hombres. 10
\end{abstract}

Die von Narváez geleitete Expedition wird bald schon zu einem völligen Desaster. Weder entdecken die Spanier wie wenige Jahre zuvor in Mexiko ein großes, mächtiges Reich voller Reichtümer noch gelingt es ihnen, sich in den Besitz dieses größtenteils von nomadisierenden, kriegerischen Stämmen bewohnten Landes zu bringen. Schlimmer noch: Hunger, Krankheiten, Schiffbruch und Angriffe der Indianer dezimieren die Expedition so sehr,

10 Álvar Núñez Cabeza de Vaca (1984: 41). Verweise auf diese Textausgabe finden sich im Weiteren unter Verwendung der Sigle NC sowie der entsprechenden Seite im fortlaufenden Text. 
dass von der ursprünglichen kleinen Armada nur noch ganze vier Männer überleben werden. Der Bericht von Álvar Núñez Cabeza de Vaca bietet eine in einer eindrücklichen, gleichsam Unmittelbarkeit erzeugenden Sprache verfasste Abfolge von Katastrophen, Leid und immer wieder überwundener Hoffnungslosigkeit, die wesentlich vom Kontakt mit der indigenen Bevölkerung geprägt wird:

Los indios, de ver el desastre que nos había venido y el desastre en que estábamos, con tanta desventura y miseria, se sentaron entre nosotros, y con el gran dolor y lástima que hobieron de vernos en tanta fortuna, comenzaron todos a llorar recio, y tan de verdad, que lejos de allí se podía oír, y esto les duró más de media hora; y cierto ver que estos hombres tan sin razón y tan crudos a manera de brutos, se dolían tanto de nosotros, hizo que en mí y en otros de la compañía cresciese más la pasión y la consideración de nuestra desdicha. (NC, 73)

Die Darstellung dieses indianischen Rituals tränenreicher Begrüßung, das in dieser Passage noch naiv als spontane Gefühlsregung missdeutet wird, leitet über zu einer Kette genauester Beobachtungen der unterschiedlichsten indigenen Völker und Stämme, die Álvar Núñez im weiteren Verlauf seiner Reise - falls man diese Bezeichnung für das von ihm Geschilderte überhaupt verwenden kann - kennenlernen sollte. Denn in der Tat hatte die Expedition des Adelantado nicht nur von der Bucht von Tampa aus den Norden Floridas erreicht, um dann in westlicher Richtung die heutige Bucht von Mobile und die Mündung des Mississippi zu passieren. Nach der Zerstreuung und dem Untergang des Hauptteils des Expeditionsheeres versuchen die vier Überlebenden, sich nach Westen zu dem ihnen gerüchteweise bekannten Südmeer und schließlich dem von Cortés eroberten Reich der Azteken durchzuschlagen. Ein Marsch über Tausende von Kilometern beginnt, der die kleine Gruppe zäh an ihre Errettung glaubender Männer vom Mississippi-Delta quer durch den Süden und Südwesten Nordamerikas bis ins heutige Kalifornien führt, von wo aus sie sich nach Süden wendend dann die von Cortés eroberten Gebiete und schließlich México, die Hauptstadt des neu gegründeten Reiches, im Jahre 1535 erreichen, wo ihnen ein triumphaler Empfang zuteil wird. Erst im August 1537 kehrt Álvar Núñez Cabeza de Vaca - den später kaum weniger gefährliche Abenteuer im Süden des Kontinents, in Asunción und entlang des Río Paraná erwarten wieder auf iberischen Boden zurück, wo sein Bericht zwischen 1537 und 1540 entsteht.

Die Durchquerung des gesamten Südens der heutigen Vereinigten Staaten, von Florida bis Kalifornien, führt Álvar Núñez durch unterschiedlichs- 
te Klimazonen und Landschaftsformationen, macht ihn mit den verschiedensten indianischen Kulturen und Stämmen bekannt ${ }^{11}$, lässt ihn, dem Amerika zuvor völlig unbekannt geblieben war, nicht aus der Rolle des Eroberers, sondern aus jener des nackten, allen Schicksalsschlägen Ausgelieferten die Riten und Gebräuche, Anbaumethoden und Glaubensformen der amerikanischen Bevölkerung jenes riesigen Landstrichs erleben. Für die Erforschung der oftmals schon im 18. Jahrhundert größtenteils dezimierten und im weiteren Verlauf der nordamerikanischen Geschichte überwiegend ausgelöschten indigenen Bevölkerung ist der überraschend präzise Bericht des Spaniers trotz seiner Einfügung fiktionaler Elemente noch heute als ethnographische Quelle von größter Bedeutung. Zugleich verschafft uns der Bericht Einsicht in die Grunderfahrung einer schier unendlichen Größe und Weite des Landes, die in Cortés' Text bereits anklang, bei Núñez Cabeza de Vaca aber zu einer fast unvorstellbaren Erfahrung des Weges von coast to coast wird. So schreibt er im 31. Kapitel, das uns auch Einblick in die Gegensätze zwischen nomadisierenden und Ackerbau treibenden indianischen Völkern gewährt, unter dem Titel »De cómo seguimos el camino del maíz«:

\footnotetext{
Pasados dos días que allí estuvimos, determinamos de ir a buscar el maíz, y no quisimos seguir el camino de las Vacas, porque es hacia el Norte, y esto era para nosotros muy gran rodeo, porque siempre tuvimos por cierto que yendo la puesta del sol habíamos de hallar lo que deseábamos; y ansí, seguimos nuestro camino, y atravesamos toda la tierra hasta salir a la mar del Sur [...]. (NC, 123)
}

Die Erfahrung des Raumes und die beständige Bewegung, das Bewusstsein eines Weges nach Westen in einem Gebiet, dessen Küstenlinie Juan de la Cosa ein gutes Vierteljahrhundert zuvor kaum mehr als erahnen ließ, und das den Spaniern völlig unbekannt geblieben war, bilden die Grundbestandteile eines Textes, der im Kontext weiterer gescheiterter Expeditionen ein Verständnis dafür vermittelt, warum nicht nur der nach Süden deutende Flug der Vögel den Spaniern die Hauptrichtung ihrer Eroberungen und Kolonisationsbemühungen vorgab. Die Naufragios markieren eine Grenzerfahrung ebenso im existenziellen wie im kulturellen und räumlich-territorialen Sinne. ${ }^{12}$ Auch bei Álvar Núñez Cabeza de Vaca wird der Weg Rich-

11 Cf. hierzu auch Roberto Ferrando (1984).

12 So ließe sich vielleicht die etwas überspitzende Formulierung von Emir Rodríguez Monegal verstehen, der in Bezug auf die Naufragios schrieb: »Diese Spanier, die so stolz auf ihren Glauben und die 
tung Norden verlassen, erst der Schwenk nach Süden bringt schließlich die Rettung auf neuspanisches Gebiet. Die Naufragios zeigten bald nicht mehr nur den Lesern in der Real Audiencia del Consejo de Indias, sondern auch einer breiteren Leserschaft in Spanien auf, wie schnell die amerikanischen Träume in Alpträume umschlagen konnten. Und sie vermittelten zugleich ein Gefühl für die fast unbegrenzte Ausdehnung eines Raumes, in dem der Autor dieses >Schiffbruchs mit Zuschauer`zwischen 1527 und 1535 umherirrte.

II.

Im Rahmen dieser kurzen Darstellung ist es nicht möglich, den weiteren Verlauf der gerade auch im Bereich der Karibik, also der `Schaltstelle $<$ zwischen Nord und Süd in Amerika, überaus komplizierten Kolonialgeschichte aufzuzeigen. Sie führte letztlich zu einer Vertiefung jenes Gegensatzes zwischen den sbeiden Amerikas und zur Errichtung jener symbolischen, aber darum nicht weniger materiellen Grenze, die den Süden der USA und Mexiko voneinander trennt und zugleich miteinander verbindet. Der Weg von Álvar Núñez Cabeza de Vaca zeigte noch bei allen kulturellen Differenzen die Kontinuität der indigenen Völker jenes Raumes auf, der heute diesseits und jenseits der politischen, aber auch der von den mit Amerika befassten Disziplinen errichteten Grenzen liegt. Was mit dem Flug der Vögel begann, war bereits im 18. Jahrhundert fest in den Köpfen der Europäer verankert. Ganz selbstverständlich zieht Guillaume-Thomas Raynal in seiner enzyklopädistischen Traditionen folgenden Darstellung der kolonialen Expansion Europas, in seiner erstmals 1770 erschienenen und in der Folge noch wesentlich erweiterten Histoire des deux Indes, eine nicht nur geographisch begründete Grenze zwischen den spanischen und portugiesischen Kolonien im Süden und den sich konstituierenden Vereinigten Staaten im Norden des Kontinents. So heißt es im achtzehnten, den (ehemaligen) englischen Kolonien in Nordamerika gewidmeten Buch der dritten Ausgabe in einer Passage, die aus der Feder von Denis Diderot stammt:

Macht des Reiches waren, das sie repräsentierten, kamen voll ausgerüstet und prächtig gekleidet an, um von dem riesigen, unbekannten Land Besitz zu ergreifen. Plötzlich versetzten das Toben des Meeres und die Unfruchtbarkeit des Landes sie zurück in das Stadium ursprünglicher Nacktheit Jahrhunderte der Zivilisation werden innerhalb von Stunden zunichte«, Emir Rodríguez Monegal (1982: 19). 
Par un contraste singulier avec l'ancien monde, où les arts sont allés du Midi vers le Nord, on verra dans le nouveau le Nord éclairer le Midi. Jusqu'à nos jours, l'esprit a paru s'énerver comme le corps dans les Indes Occidentales. Vifs \& pénétrans de bonne heure, les hommes y conçoivent promptement: mais n'y résistent pas, ne s'y accoutument pas aux longues méditations. Presque tous ont de la facilité pour tout; aucun ne marque un talent décidé pour rien. Précoces \& mûrs avant nous, ils sont bien loins de la carrière quand nous touchons au terme. ${ }^{13}$

Aufschlussreich ist hier nicht nur die schematische Gegenüberstellung von Norden und Süden, die mit der im 18. Jahrhundert verbreiteten These von einer Körper und Geist erfassenden Degradation gekoppelt wird, so dass der gegenüber den Indianern seit Beginn der Conquista geäußerte IndolenzVorwurf nun auf alle Bewohner des südlichen Amerika, auch jene europäischer Herkunft, ausgedehnt werden kann. Bemerkenswert ist auch die Tatsache, dass im individuellen wie im kollektiven Bereich eine andere Entwicklungskurve angenommen wird, wobei Diderot das nous seiner impliziten europäischen Leserschaft in eine identifikatorische Beziehung zum Norden des amerikanischen Kontinents bringt. Stellten die spanischen Eroberer im 16. Jahrhundert fest, dass sich die wesentlich komplexeren, entwickelteren indianischen Gesellschaften allesamt im Süden des Kontinents befanden, so dreht sich die Einschätzung im 18. Jahrhundert so, dass im Verlauf des Siècle des Lumières, in dem Spanien und alles Spanische kulturgeschichtlich wie philosophisch in eine Randstellung gedrängt wurde ${ }^{14}$, die politische, kulturgeschichtliche und künstlerische Bewertung mehr oder minder vollkommen umschlug. Bereits der obige Textauszug macht deutlich, dass Nordamerika nun in eine überlegene Position gerückt war, dass den Vereinigten Staaten kraft ihrer angenommenen >Superiorität und ihrer stetigen Entwicklung zunehmend Modellcharakter für den gesamten Kontinent (und später - wie wir wissen - weit darüber hinaus) zukam. Gab es auch im Verlauf der beiden zurückliegenden Jahrhunderte Gegenstimmen sowohl von europäischer als auch von spanischer, hispanoamerikanischer und später panlatinistischer Seite, so blieben die USA doch spätestens seit Hegel unverrückbar in der geschichtsphilosophisch abgesicherten Position der Superiorität. Die machtpolitische Expansion der USA seit Ausgang des

13 Guillaume-Thomas Raynal (1781: 108).

14 Charakteristisch und besonders anschaulich ist - gewiss neben vielen anderen Zeugnissen - der Artikel »Espagne« von Nicolas Masson de Morvilliers in der Encyclopédie méthodique. Géographie (1783: Bd. 1, 554-568). 
19. Jahrhunderts tat ein Übriges. Im Licht, das die Vereinigten Staaten von Amerika in der Histoire des deux Indes dem Süden bringen sollten, verschwanden die anderen Regionen des amerikanischen Kontinents nahezu vollständig oder dienten doch nur mehr als Negativfolie für den scheinbar kometenhaften Aufstieg der Macht im Norden. Die amerikanischen Träume wurden fortan zunehmend nach Norden projiziert. Schon in seinem auf das Jahr 1896 datierten kulturkritischen Essay Ideárium español, das zur Bibel der spanischen Generación del 98 werden sollte, musste der an spanischen Konsulaten im Ausland arbeitende Ángel Ganivet feststellen, dass die USA längst zu einer »nación formidable « geworden war, die ihre »protección paternalista « über ganz Amerika ausdehne und sich bereits in die Angelegenheiten Europas einmische. Überall in Europa verstehe man unter der Bezeichnung >Amerika nur mehr die Vereinigten Staaten, ein >Amerikaner sei selbstverständlich nur ein Bürger der USA, während man bei Angehörigen der hispanoamerikanischen Staaten stets den Namen des jeweiligen Landes hinzusetzen müsse. ${ }^{15}$ Dass sich hieran bis heute nichts Wesentliches geändert hat, ist leicht festzustellen; doch fällt es schwer zu akzeptieren, dass selbst akademische Disziplinen derart unpräzisen umgangssprachlichen Wendungen die höheren wissenschaftlichen Weihen gaben, so dass man bis heute unter der Bezeichnung >Amerikanistik etwa im Bereich der Anglistik völlig selbstverständlich wenig mehr als eine ,Vereinigte-Staaten-von-Nordamerikanistik praktiziert. Nicht ganz zu Unrecht wird die deutsche Universität noch heute als eine Schöpfung Humboldts verstanden, doch ist damit nur Alexanders Bruder Wilhelm gemeint, der Europa bekanntlich nie verließ.

Im melting pot dieser Vorstellungen droht die kulturelle Vielgestaltigkeit Amerikas - also des gesamten Kontinents - bisweilen unterzugehen. Es ist dabei nicht uninteressant, dass ein kritischer Leser Raynals, aber auch Hegels, Alexander von Humboldt, eigene Vorstellungen hierzu entwickelte. Er hatte auf seiner Reise zwischen 1799 und 1804 zusammen mit Aimé Bonpland mit Erlaubnis des Katholischen Königs die spanischen Kolonien in Amerika bereist und auf diese Regionen sein Hauptaugenmerk gerichtet. Doch ließ ihn seine allgegenwärtige Methode des Vergleichs oftmals auch Nordamerika miteinbeziehen. Besuchte er die USA am Ende seiner Reise auch nur kurz und vor allem, weil er durch eine Rückreise über

15 Ángel Ganivet (1961: 245). 
die Vereinigten Staaten sicherstellen wollte, dass ihm spanische Behörden nicht die Ergebnisse seiner Forschungen rauben und eventuelle Publikationen über das spanische Kolonialreich unterdrücken konnten ${ }^{16}$, so verhinderte dies weder seine Auseinandersetzung mit der großen Republik im Norden noch die Tatsache, dass er im weiteren Verlauf des 19. Jahrhunderts in den USA zu einem wahren Kulturheroen avancieren konnte. Im dritten Band seiner Relation historique, in den auch vielfältige statistische Untersuchungen zum Verhältnis zwischen dem Norden und Süden Amerikas Eingang fanden, schrieb er unter dem Einfluss der Unabhängigkeitskriege - »une de ces grandes révolutions qui agitent de temps en temps l'espèce humaine« - in den von Spanien abgefallenen Kolonien:

Aujourd'hui, la partie continentale du Nouveau-Monde se trouve comme partagée entre trois peuples d'origine européenne: l'un, et le plus puissant, est de race germanique; les deux autres appartiennent, par leur langue, leur littérature et leurs mœurs, à l'Europe latine. ${ }^{17}$

Diese Aufteilung, die in gewisser Weise den im Zeichen eines unter französischer Hegemonie stehenden Panlatinismus entwickelnden Terminus $>$ Lateinamerika ${ }^{18}$ mit seiner vorwiegend kulturellen Fundierung vorwegnimmt, übersieht dabei nicht, dass es ein Amerika der Indianer, ein Amerika der Schwarzen - gerade den »Africains libres d'Haïti« widmete er besondere Aufmerksamkeit (RH, 57) -, ein Amerika von Kolonisten slawischer (ibid.) und anderer Herkunft gab. Gleichwohl sah auch er die Trennung zwischen einem Amerika, das aus der Kolonisierung durch Völker des lateinischen Europa hervorging, und einem anderen Amerika, das er auch als das der »Anglo-Américains« bezeichnete (ibid.). ${ }^{19}$ Gewaltige Entwicklungsmöglichkeiten sieht Humboldt für alle Gesellschaften und Gebiete des amerikanischen Kontinents, doch hegt er bereits im dritten Jahr-

16 Diese Angst war aufgrund von Präzedenzfällen im spanischen Bereich nicht aus der Luft gegriffen. Die portugiesische Krone hatte Order gegeben, Humboldt - sollte er das heutige Brasilien betreten sofort festzunehmen; und England hütete sich später wohl, das eigene Kolonialreich den neugierigen kritischen Augen (und der spitzen Feder) des preußischen Gelehrten zu öffnen.

17 Alexander von Humboldt (1970: 56); Verweise auf diese Textausgabe erscheinen unter Verwendung der Sigle RH sowie der entsprechenden Seite im fortlaufenden Text.

18 Cf. etwa John Leddy Phelan (1968), Joseph Jurt (1982) sowie Miguel Rojas Mix (1986).

19 Bezüglich der Trennung zwischen englischem, spanischem und portugiesischem Amerika fährt Humboldt fort: "La première de ces trois nations, les Anglo-Américains, est aussi, après les Anglois de l'Europe, celle qui couvre de son pavillon la plus grande étendue des mers. Sans colonies lointaines, leur commerce a pris un accroissement que n'a pu atteindre aucun peuple de l'ancien monde, si ce n'est celui qui a communiqué, au nord de l'Amérique, sa langue, l'éclat de sa littérature, son amour du travail, son penchant pour la liberté, et une partie de ses institutions civiles." 
zehnt des 19. Jahrhunderts aufgrund seiner Forschungen keine Zweifel mehr an der künftigen Expansion des bereits zum damaligen Zeitpunkt materiell zunehmend überlegenen Nordens. Gleichwohl hütete er sich mit guten Gründen davor, Amerika mit den USA gleichzusetzen. Seine amerikanischen Träume blieben nie auf den Norden beschränkt.

III.

In seinem berühmten, auf Salamanca im »año de gracia 1912《 datierten philosophischen Essay Del sentimiento trágico de la vida betonte Miguel de Unamuno, dass heute, im damals noch 20. Jahrhundert, alle vergangenen Jahrhunderte fortbestehen: »Subsisten hoy, en el siglo XX, todos los siglos pasados y todos ellos vivos. $\ll^{20}$ Und der spanische Essayist, Kritiker und Philosoph fügte hinzu:

Nada se pierde, nada pasa del todo, pues que todo se perpetúa de una manera o de otra, y todo, luego de pasar por el tiempo, vuelve a la eternidad. Tiene el mundo temporal raíces en la eternidad, y allí está junto el ayer con el hoy y el mañana. Ante nosotros pasan las escenas como en un cinematógrafo, pero la cinta permanece una y entera más allá del tiempo. (STV, 910-911)

Die Kopräsenz aller Jahrhunderte im 20. Jahrhundert, die Speicherung aller >lebendigen Bilder und Szenen im Medium und der Metapher des Kinematographen, mögen uns in den Worten Unamunos einen Vorgeschmack von Postmoderne für unser gerade zu Ende gegangenes Jahrhundert geben (zumal sich manch unerkannte Beziehung von diesem Essay zu den Ficciones von Jorge Luis Borges ziehen ließe). Gleichviel, das >Aufgehobensein^vorgängiger Zeiten und Bilder lässt sich auch auf jene französischen Texte und amerikanischen Träume beziehen, denen nun unsere Aufmerksamkeit gelten soll.

Michel Butors 1962 veröffentlichter Text Mobile fällt zeitlich in die Blütezeit französischer Theoriebildung. Nicht von ungefähr zählt Butor zu jenen Autoren, die nicht nur durch ihre literarischen Texte, sondern auch durch eine Vielzahl literaturtheoretischer Arbeiten hervorgetreten sind. Mobile ist ein experimenteller Text, in dem eine Reihe theoretischer Vorstellungen und Ansätze erprobt werden, und den man im weitesten Sinne -

20 Miguel de Unamuno ( $\left.{ }^{7} 1967: 844\right)$; Verweise auf diese Textausgabe werden im Weiteren unter Verwendung der Sigle STV sowie der entsprechenden Seite im fortlaufenden Text angegeben. 
nicht aufgrund der Vielzahl an Reisen, die der Autor in jenen Jahren selbst unternahm - der Reiseliteratur zuordnen oder doch von ihr aus perspektivieren könnte. Traditionelle reiseliterarische Schemata werden freilich bewusst unterlaufen, wenn auch dieser $>$ Reisebericht $\measuredangle$ als ein im Raum entfaltetes Verstehensmodell aufgefasst und gelesen werden kann. ${ }^{21}$ Mobile stellt eine Reise durch die verschiedenen Staaten der USA, durch unterschiedliche Ethnien, Kulturen und Daseinsformen in jenem Land dar, das spätestens seit Ende des Zweiten Weltkriegs für die Europäer zu einem Mythos und zur Verkörperung dessen geworden war, was man den American Dream nannte. Viele der auf den Norden Amerikas projizierten Träume und Mythen sind in den Text integriert, von der schon von den Spaniern im 16. Jahrhundert gesuchten Nordwestpassage als Durchfahrt zum Pazifik (deren angenommene Existenz noch einen Chateaubriand faszinierte ${ }^{22}$ ) bis hin zur automobilen Freiheit unbeschränkter Beweglichkeit, die nicht nur durch die vielfach inszenierten Automarken und Bewegungsformen, sondern auch durch den Titel des Textes selbst aufgerufen wird. Denn der Titel Mobile zeichnet den Band von Beginn an als polysemen Bewegungstext aus: Konnotiert werden - um nur einige grundlegende, mit Hilfe des Titels aufgerufene Isotopien anzusprechen - die ständige, unabschließbare Bewegung, die bereits Butors frühen Texten, die stets auf einem mobilen Grundschema beruhten, das die Aktivität des Lesers herausforderte, eigen war; die Stadt im Süden der USA, an deren Bucht lange vor ihrer Gründung Álvar Núñez Cabeza de Vaca vorbeigezogen war; der Name eines Konzerns, der mit Treibstoffen Bewegungsmittel feilbietet, die im Text selbst immer wieder getankt werden; und schließlich ein Mobile, also ein sich ständig in Bewegung befindliches kunsthandwerkliches oder künstlerisches Produkt, das mit seinen beweglichen Einzelteilen zwischen diesen und im Verhältnis zu deren Umfeldern unablässig neue Relationen und Konstellationen aufbaut. Schon den jungen Butor hatten die Mobiles, die beweglichen Skulpturen des nordamerikanischen Künstlers Alexander Calder beeindruckt, die für seinen Text von 1962 quasi Modellcharakter annehmen. Angesichts der seit den gerade in den sechziger Jahren engen Verschränkung zwischen Literatur und Theoriebildung erstaunt es nicht, dass

21 Cf. hierzu allgemein Ottmar Ette (1997).

22 Selbstverständlich fehlt auch der Traum vom Fernen Westen, von San Francisco, ebenso wenig wie der von El Dorado; beide werden von Beginn an in den Text eingefügt; cf. Michel Butor (1991: 2425); im Folgenden wird auf diese Textausgabe unter Verwendung der Sigle M verwiesen. 
in einem ebenfalls 1962 veröffentlichten Buch des italienischen Zeichentheoretikers Umberto Eco nicht zuletzt die Mobiles Calders jene ästhetische Konzeption repräsentieren, die Eco als die des >offenen Kunstwerks bezeichnete. ${ }^{23}$ Butors Mobile ist in diesem Sinne ein offenes Kunstwerk, eine opera in movimento.

Butors Experimentaltext trägt nicht umsonst den Untertitel »Étude pour une représentation des États-Unis«. Hatte Butor seit den fünfziger Jahren verschiedenste Mythen abendländischer Tradition in seinen Texten verarbeitet, so greift er hier - und Roland Barthes' »Mythen des Alltags $\ll^{24}$ sind nicht fern - den Mythos Amerika, den in den USA verkörperten Mythos der Moderne schlechthin auf. Schon in dem an Werbetexte von Reiseveranstaltern angelehnten Klappentext werden die USA als ganzer Kontinent, als Ort einer gewaltigen Natur, aber auch als Raum von vielfältigster Erfahrung, von Abenteuer und Unendlichkeit präsentiert, was vor den angesprochenen französischen beziehungsweise europäischen Lesern alle historisch akkumulierten Bilder und Vorstellungen ganz bewusst Revue passieren lässt. Als relationale und serielle Grundstruktur des Grenz-Textes (œuvrelimite) fungiert ein rational kontrolliertes Springen ${ }^{25}$ von Bundesstaat zu Bundesstaat innerhalb der Grenzen der Vereinigten Staaten, wobei dem Phänomen der Grenze und der Grenzerfahrung von Beginn an große Bedeutung beigemessen werden. Nicht nur die politischen Außengrenzen, sondern auch Binnengrenzen und mehr noch Binnenausgrenzungen werden markiert: etwa mit Hilfe des wiederholten Hinweises auf Reservate von Indianern (M, 24 und passim), die im übrigen fortwährend kulturelle Alteritätserfahrung in den Text integrieren, oder durch die Einblendung von Hinweisschildern, welche die Schwarzen in den Bussen ausgrenzen (M, 27). Die historische Dimension von Segregation und Genozid wird immer wieder am Beispiel der verschiedenen indianischen Stämme und Völker vorexerziert: In allen Staaten wird auf diese ethnischen Gruppen verwiesen, auch wenn sie zumeist längst ausgelöscht wurden, so dass bisweilen nur

23 Cf. Umberto Eco (1976: 157): „Calder fa un passo avanti: ora la forma si muove essa stessa sotto i nostri occhi, e l'opera diventa >opera in movimento<. Il suo movimento si compone con quello dello spettatore. A rigore non dovrebbero esservi mai due momenti, nel tempo, in cui la posizione reciproca dell'opera e dello spettatore possano riprodursi in modo uguale. Il campo delle scelte non è piú suggerito, è reale e l'opera è un campo di possibilità.«

24 Cf. Roland Barthes (1957).

25 Dies bedeutet, dass narrative und diskursive Kontinuitäten immer wieder unterlaufen, zugleich aber markiert werden. Auf die Problematik des Diskontinuierlichen in Butors Text hatte in einem bereits 1962 verfassten Essay Roland Barthes (1964) hingewiesen. 
mehr auf die - bereits von Álvar Núñez beschriebenen - Tumuli der Begräbnishügel aufmerksam gemacht werden kann (ibid.). ${ }^{26}$ Die Absenz der ursprünglichen amerikanischen Bevölkerung wird so in Mobile in eine ständig wieder aufgerufene Präsenz verwandelt.

Mit der Erfahrung dieser Binnengrenzen paradox gekoppelt ist freilich stets die Erfahrung naturräumlicher Unbegrenztheit in der diskontinuierlichen, unabschließbaren Bewegung zwischen den unterschiedlichsten Elementen. In Butors Mobile erscheinen die Vereinigten Staaten zwar nicht als Land der unbegrenzten Möglichkeiten, wohl aber als Land einer unbegrenzten Kombinatorik, deren Spielcharakter aus der europäischen Distanz durch die mise en page im Querformat sichtbar und anhand des Sprachmaterials für den Leser erfahrbar gemacht wird. Die Verbindung unterschiedlichster Diskursfragmente führt - ganz im Sinne Unamunos - zu einer lebendigen Vergleichzeitigung aller historisch akkumulierten Traumbilder, deren imaginäre wie kinematographische Projektionsfläche die Vereinigten Staaten beziehungsweise Amerika waren.

In einer Reihe von Publikationen, die sich mit außereuropäischen Kulturen und speziell mittel- und nordamerikanischen Indianern beschäftigen, zu denen er seit den sechziger Jahren direkte Kontakte pflegte, hat JeanMarie Gustave Le Clézio die Herausforderung kultureller Alteritätserfahrung künstlerisch wie kulturtheoretisch fruchtbar gemacht. 1988 erschien Le rêve mexicain, ein Buch, das sich unter anderem gestützt auf die Historia verdadera de la conquista de la Nueva España des Bernal Díaz del Castillo, eines Soldaten von Hernán Cortés bei der Eroberung Mexikos, mit jenem Traum beschäftigt, der zwar ein amerikanischer Traum ist, aber nicht als American Dream missverstanden werden will und so - ganz im Sinne Ganivets - der nationalen Spezifizierung bedarf. Vom Traum der Eroberer bis hin zum Traum der französischen Surrealisten, die wie Antonin Artaud Mexiko aufsuchten, letztlich aber auch von seinem eigenen AmerikaTraum, ist in Le Clézios Buch die Rede. Schon der Klappentext dieses Bandes stellte die Kernfrage des französischen Autors:

26 Immer wieder werden Fetzen einer Geschichte interkultureller Missverständnisse, von Kulturzusammenstoß und Genozid, eingeblendet (besonders eindrücklich etwa M, 124ss.). 
Le rêve mexicain, c'est cette question aussi que notre civilisation actuelle rend plus urgente: qu'aurait été notre monde, s'il n'y avait eu cette destruction, ce silence des peuples indiens? Si la violence du monde moderne n'avait pas aboli cette magie, cette lumière? ${ }^{27}$

Die Zerstörungswut europäischer Moderne seit der Conquista ist zugleich Folge und Bedingung jener nach Amerika projizierten Träume, die in Mexiko mit seiner konstitutiven Präsenz indigener Bevölkerung wie in einem Brennspiegel zusammenzulaufen scheinen. Das kulturell Andere, das schon im Untertitel als unterbrochen aber nicht abgebrochen apostrophierte Denken der Indianer, wird dabei zur Herausforderung des abendländisch geprägten Menschen, zur Verheißung einer Wiederkehr des Verdrängten, Ausgegrenzten, Vernichteten, zu dem an diesem Ort noch Zugänge wiederauffindbar zu sein scheinen. Der >mexikanische Traum widersprüchlich, enthält er doch für Le Clézio von Beginn an nicht nur die Träume von Eroberung, Gold und Reichtum, sondern auch jene der Azteken von einer Wiederkehr Quetzalcóatls und einer Auslöschung ihres eigenen Reiches. In diesem kultur- und zivilisationskritischen ${ }^{28}$, oftmals sehr manichäistisch strukturierenden Text wird daher der Funktion der Träume in den indianischen Kulturen eine wichtige Rolle zugewiesen (RM, 169ss.). Der im Buch dargestellte gnadenlose Exterminationskrieg der Spanier, der bereits auf jenen der Nordamerikaner im 19. Jahrhundert vorausweise, konnte freilich den auch über unsere Zeit fortbestehenden smexikanischen Traum<, an die Ursprünge der Zivilisation zurückkehren zu können, in seiner Existenz nicht gefährden. Mit den Mitteln von Anthropologie und Literatur versucht Le Clézio, an den unterbrochenen Traum wieder anzuknüpfen, dazu beizutragen, dass auch diese Elemente vergangener Jahrhunderte und Kulturen nicht gänzlich verschwinden, sondern im 20. Jahrhundert in lebendige kulturelle Formen überführt werden können. Der

27 Jean-Marie Gustave Le Clézio (1988: Klappentext); die Textausgabe wird unter der Sigle RM zitiert.

28 Dabei geht es immer wieder um das europäische Projekt der Moderne, das als ein Mechanismus sukzessiver Ausgrenzungen erscheint: »La Conquête n'est pas seulement la mainmise d'une poignée d'hommes - étrange mélange de barbarie et d'audace - sur des terres, des réserves alimentaires, des routes, des organisations politiques, sur la force de travail des hommes et la réserve génétique des femmes. Elle est la mise en œuvre d'un projet conçu à l'origine même de la Renaissance, en vue de la domination du monde. Rien de ce qui fut le passé et la gloire des nations indigènes ne doit survivre: la religion, les légendes, les coutumes, l'organisation familiale ou tribale, les arts, le langage, et jusqu'à l'histoire, tout doit disparaître afin de laisser la place au moule nouveau imposé par l'Europe« (RM, 209). 
Traum geht also weiter. Le Clézios Traum ist letztlich ein Projekt einer Prämoderne, die in die Schule der Moderne gegangen ist und sich deshalb sehr wohl mit den Vergleichzeitigungsprozessen der Postmoderne in Verbindung bringen lässt.

Schon Michel Butor räumte in seinem literarischen USA-Mobile gerade den Landschaften des Südens und Südwestens und dabei wiederum den Wüsten einen besonderen Stellenwert ein. Für eine Vielzahl europäischer wie amerikanischer Schriftsteller sind in der Tat Wüstenlandschaften zu zentralen Schauplätzen von Romanen, Erzählungen und Reiseberichten geworden, wobei gerade die Autoren unter postmodernem Vorzeichen sich gerne dieser ariden Landschaftsformationen bedienen. An die Stelle der Fülle treten Kargheit und Leere, eine Leere freilich, die nicht nur in besonderer Weise das Individuum mit sich selbst konfrontiert und damit die Auseinandersetzung mit individuellen Identitätsbildungsprozessen intensiviert, sondern zugleich auch auf kollektiver Ebene Identitätskonstruktionen herausfordert und mehr noch neue Projektionsflächen - nicht zuletzt für neue amerikanische Träume - bietet. Als eindrückliches Beispiel hierfür kann auch ein Text des französischen Philosophen und Kulturtheoretikers Jean Baudrillard gelten, wobei wir uns vorab der Frage stellen sollten, welche Funktionen der Wüste innerhalb der Abfolge amerikanischer Traumbilder zukamen.

In seinem kurzen, ursprünglich wohl 1807 entstandenen und 1808 in die Erstausgabe seiner nicht nur vom deutschsprachigen, sondern auch vom europäischen Publikum insgesamt wohlwollend bis begeistert aufgenommenen Ansichten der Natur integrierten Text »Über die Steppen und Wüsten« schrieb Alexander von Humboldt:

Denn wenn im raschen Aufsteigen und Niedersinken die leitenden Gestirne den Saum der Ebene erleuchten; oder wenn sie zitternd ihr Bild verdoppeln, in der untern Schicht der wogenden Dünste, glaubt man den küstenlosen Ozean vor sich zu sehen. Wie dieser erfüllt die Steppe das Gemüth mit dem Gefühl der Unendlichkeit. Aber freundlich zugleich ist der Anblick des klaren Meeresspiegels, in dem sich die leichtbewegliche sanft aufschäumende Welle kräuselt. Todt und starr liegt die Steppe hingestreckt, wie die nackte Felsrinde eines verödeten Planeten. $^{29^{\circ}}$

29 Alexander von Humboldt (1808: 2-3). In einer Anmerkung zu dieser Passage wird in bewegten Worten der »unauslöschliche« Eindruck hervorgehoben, den die Llanos, von denen hier die Rede ist, aus dem Kontrast zum Dickicht der Urwälder entfalteten. 
Diese Passage Alexander von Humboldts, der - so weit ich sehe - erstmals die Andenlandschaften ästhetisch behandelte und damit eine Pionierfunktion übernahm, die bislang wenig ins Blickfeld gerückt wurde, bildet eine wichtige Phase des Übergangs zu dem sich weitaus später, dominant erst in der zweiten Hälfte des 20. Jahrhunderts vollziehenden Paradigmenwechsel von der Fülle zur Kargheit und (Menschen-)Leere. Die unmittelbare Assoziation von Unendlichkeit, eines schier unbegrenzten und zugleich (scheinbar) menschenleeren Raumes, der sich dem Blick des Individuums darbietet, bringt Steppe und Wüste in einen direkten Vergleich mit der Fläche des Ozeans. Beide berühren sie unvermittelt das Gemüt des Menschen, verwandeln sich in Seelenlandschaften. Ozean wie Steppe vermitteln - wie Humboldt dieser Passage später hinzufügt - mit den »Eindrücken des Raumes« zugleich geistige »Anregungen höherer Ordnung « ${ }^{30}$. Die Flächen von Meer und Steppe sind gleichsam philosophische Landschaften, die größten Einfluss auf ihren Betrachter ausüben, auch wenn der Ozean den freundlicheren Anblick bietet. Steppe und Wüste, Bilder eines »verödeten Planeten«, sind ebenso wie das Meer menschenleere Räume, die zur Durchquerung und damit zu räumlicher wie geistiger Bewegung auffordern. Sie wollen befahren und zugleich erfahren werden, da sie nur so ihr Rätsel preisgeben. Als in Europa nicht in dieser Ausdehnung vorhandene landschaftliche Ausdrucksformen der Anökumene, in welcher der (abendländische) Mensch nicht dauerhaft wohnen kann, bieten sie Raum für die Erfahrung der Grenze wie für Grenzerfahrungen. Erst durch diese Erfahrung in der Bewegung verwandelte Kolumbus das Meer aus dem trennenden in ein verbindendes Element. Die dem Blick preisgegebene Fläche vermittelt ein Gefühl für die Ausdehnung und Existenz unseres Planeten. So ist es paradoxerweise die sich der dritten Dimension verweigernde Oberfläche, die mit ihrer Wölbung die Kugelform des Planeten - wie einst bei der ersten Fahrt des Kolumbus - immer wieder ins Bewusstsein ruft. ${ }^{31}$

So ist die unendliche, scheinbar menschenleere Fläche aufs Engste mit der Entdeckung wie der Eroberung, aber auch (wie etwa bei Álvar Núñez Cabeza de Vaca) mit der Erfahrung des Sich-Verlierens und Behauptens in dieser unermesslichen Weite verbunden und tief mit dem auf Amerika be-

30 Cf. den schönen Wiederabdruck der dritten, von Humboldt 1849 mit einem neuen Vorwort versehenen Ausgabe der Ansichten der Natur. Nördlingen: Greno, 1986, p. 16.

31 In Humboldts Anmerkungen liest man: »Die Sonne war eben untergegangen. Die Steppe schien wie eine Halbkugel anzusteigen«, Alexander von Humboldt (1986: 44). 
zogenen kollektiven Imaginären des Europäers verknüpft. Dies mag auch eine kurze, abschließende Beschäftigung mit Amérique von Jean Baudrillard aufzeigen.

Das erstmals 1986 erschienene Bändchen widmet sich unter dem kontinentalen Titel ganz selbstverständlich nur den USA, die - der Diskurs von Baudrillards Landsmann Alexis de Tocqueville in De la démocratie en Amérique hinterließ unverkennbar seine Spuren - gleichsam als das Modell einer globalen Entwicklung erscheinen, die in Europa bislang höchstens als Abglanz oder mehr oder minder schlechte Synchronisierung eines in den Vereinigten Staaten hervorgebrachten Streifens erscheinen. ${ }^{32}$ Die im Buch dieses französischen (Vor-)Denkers der Postmoderne erscheinenden Globalisierungsprozesse sind, wie schon die semantische Reduktion des Titels auf die USA nahe legt, wenig reflektiert und bestenfalls auf eine G-7-Problematik reduziert. Die dem Buch vorangestellte Warnung »Caution: objects in this mirror may be closer than they appear « (A, 7) führt spielerisch und elegant verschiedene für den Text wichtige Bedeutungsebenen ein. Die Warnung verweist zum einen auf die in den USA gesetzlich vorgeschriebene Aufschrift auf verkleinernden Rückspiegeln nordamerikanischer Automobile, womit nicht nur das beliebteste Fortbewegungsmittel der Nordamerikaner, sondern auch ein zentrales Erfahrungs-Mittel des Buches eingeführt wird. Und zum anderen wird ingeniös die Spiegelmetapher in dieses Beispiel einer postmodernen Reiseliteratur eingeblendet, wobei ganz im Stendhalschen Sinne nicht der Autor, sondern die im Buch gespiegelte Realität für das den Leser vielleicht Bedrückende - und ihm schon nah auf den Leib Gerückte - verantwortlich gemacht wird. In der Tat wird nicht nur Stendhals, sondern auch Baudrillards Spiegel eine Straße entlang geführt, mehr noch: eine schier unendliche Vielzahl an highways und freeways, von denen aus der Erzähler seinem Leser die Vereinigten Staaten präsentiert. Wieder wird Amerika damit aus der Bewegung erfahren, wie schon bei Butor freilich nicht mehr aus einer zielgerichteten und logischen Bewegung heraus, sondern in einer akausalen Abfolge sich diskontinuierlich einander abwechselnder Autobahnstücke. Mit dem Spiegel verbindet sich zugleich aber eine Überwindung eben des Mimetischen, insoweit diese Welt nun als

32 Jean Baudrillard (1986: 76): "L'Amérique est la version originale de la modernité, nous sommes la version doublée ou sous-titrée. « Diese Textausgabe wird im Weiteren unter Verwendung der Sigle A zitiert. 
unendliche Abfolge von Spiegelungen und damit als Simulacrum einer vorgespie(ge)lten Realität erscheint. An die Stelle sauthentischer Erfahrung tritt ein Netzwerk von Substitutionen und Erfahrungs-Surrogaten. Auch diese Konzeption Amerikas setzt eine Kombinatorik in Bewegung, in welcher sich Sinn-Bruchstücke und fragmentierte Simulacra ständig neu beleuchten. Vom incipit dieses Textes an ist der American Dream - vorwiegend in der Variante räumlicher Freiheit - und dessen mediale, stereophone wie filmische Erfahrung präsent:

\begin{abstract}
Nostalgie née de l'immensité des collines texanes et des sierras du NouveauMexique: plongées autoroutières et supertubes sur la stéréo-Chrysler et vague de chaleur - la photo ponctuelle n'y suffit plus - il faudrait avoir le film total, en temps réel, du parcours, y compris la chaleur insupportable et la musique, et se reprojeter tout cela intégralement chez soi, en chambre noire - retrouver la magie de l'autoroute et de la distance, et de l'alcool glacé dans le désert et de la vitesse, revivre tout cela au magnétoscope chez soi, en temps réel - non pour le seul plaisir du souvenir, mais parce que la fascination d'une répétition insensée est déjà là, dans l'abstraction du voyage. Le déroulement du désert est infiniment proche de l'éternité de la pellicule. (Ibid.)
\end{abstract}

Erfahrung erscheint immer schon als Spiegelung, Unermesslichkeit als Sehnsucht und Nostalgie nach der Erfahrung von Unermesslichkeit, die mediatisiert vervielfältigt wird. Das Abrollen der Wüste erscheint als Ewigkeit der akkumulierten Bilder eines Films ganz im Sinne Unamunos, zugleich lebendig und als Zeichen gespeichert und wahr-genommen. Alles in dieser Bilderfolge wirkt irgendwie vertraut, wie das déjà-vu längst bekannter Träume von Amerika, die nun zu Eis gefroren, festgebannt in Echtzeit nochmals ablaufen und Erfahrungen substituieren und zugleich bilden. Längst ist die Wirklichkeit dem Film nahe gekommen, ganz so, wie die nordamerikanischen Städte so wirken, als seien sie für ihre Mediatisierung, für ihre Fixierung in bewegten Fernsehbildern erbaut worden (A, 57). Cinecittà (oder besser: Hollywood) ist überall, die akkumulierten Träume werden abrufbar.

Die Leere und Flächenhaftigkeit der Wüste ist die Voraussetzung dafür, dass die amerikanischen Träume - wie auf die leeren Flächen der Karten von Juan de la Cosa - auf sie projiziert werden können. Voraussetzung hierfür ist die Außerhalbbefindlichkeit des Europäers und die beständige Bewegung, welche die Abfolge der Bilder - gleich der Perlenkette jener Inseln, die für Kolumbus immer gleich und immer anders waren - sicherstellt. Die ideale Projektionsfläche ist nun aber nicht mehr die überreiche, 
von einer wuchernden Vegetation bedeckte Inselwelt der Karibik, sondern die Kargheit des Südwestens, die Physiognomie der Wüste, die im Sinne Humboldts »die nackte Felsrinde eines verödeten Planeten « bietet. Auch Antonin Artaud suchte sie in der verdoppelten Darstellung von Le Clézios rêve mexicain auf, freilich noch auf der Suche nach den Ursprüngen. In Baudrillards Text wird jenseits der Refraktionen nicht mehr nach Originärem gefragt. In diesen Wüstenlandschaften der nordamerikanischen Grenzregion des Südens sind die Mexikaner längst zu Chicanos geworden und arbeiten als »guides dans la visite d'El Alamo pour exalter les héros de la nation américaine« (ibid.). Die Grenze ist durchlässig geworden, sie dient zur Bestätigung der Überlegenheit des Nordens und seiner Bilder und Traumwelten. Authentizität im borderland gibt es nicht, wohl aber deren Inszenierung.

Die Wüste zeigt in ihrer Flächenhaftigkeit, dass es nicht um ein Amerika der Tiefe, sondern um eines der Oberfläche (nicht der Oberflächlichkeit) geht, auf welcher jene Hochgeschwindigkeitsversuche (A, 12) $)^{33}$ unternommen werden, mit Hilfe derer Menschen auf dem Erdboden die größtmögliche Geschwindigkeit erreichen. Seit der Exterminierung der Wüstenindianer, die in die rasche Bilderfolge eingeblendet wird, steht dieser beschleunigten Bewegung nichts mehr im Wege: Die Felsrinde des Planeten wird sichtbar:

Il a même fallu que les Indiens en soient exterminés pour que transparaisse une antériorité encore plus grande que celle de l'anthropologie: une minéralogie, une géologie, une sidéralité, une facticité inhumaine, une sécheresse qui chasse les scrupules artificiels de la culture, un silence qui n'existe nulle part ailleurs. (A, 11)

Die Großstadt, die nordamerikanische Metropole, erscheint dann notwendig als Fortsetzung der Wüste mit urbanistischen Mitteln. Auch in ihr ist das Reich der Geschwindigkeit. Die historische Akzeleration der Moderne hat so viel Fahrt aufgenommen, dass diese Geschwindigkeit ihre eigene Zeit, ihre eigene Objektwelt und, mehr noch, ihre eigenen amerikanischen Träume herstellt. Die immense Beschleunigung hat paradoxerweise am Ende des 20. Jahrhunderts einen Stau der Bilder und der Träume Amerikas bei

33 "La vitesse est créatrice d'objets purs, elle est elle-même un objet pur, puisqu'elle efface le sol et les références territoriales, puisqu'elle remonte le cours du temps pour l'annuler, puisqu'elle va plus vite que sa propre cause et en remonte le cours pour l'anéantir.« 
jenen Europäern ausgelöst, deren Schriften hier alleine zu Wort kamen. Auch cross-cultural ausgerichtete Untersuchungen könnten jedoch sicherlich belegen, dass auch auf dem amerikanischen Kontinent selbst ein Ende der amerikanischen Träume nicht abzusehen ist. Vielleicht aber ist Amerika heute keine Utopie mehr - kein Ort, nirgends: Die Utopie scheint längst schon verwirklicht -, sondern Kernbestand jenes Bezirks, den das obige Zitat abschließend negativ umreißt: nulle part ailleurs. Wenn aber das Nirgendwo anders die Konturen des amerikanischen Traums skizziert, dann liegt der Gedanke nahe, dass die Konturen des Anderen - und damit auch Europas - wirklich verschwimmen. Die Geschichte scheint sich zu verabschieden, ein von Baudrillard freudig begrüßter Abschied. Insoweit könnte Amérique als das Bordbuch eines neuen Kolumbus gelesen werden, der in seiner Schifffahrt durch die Wüste im doppelten Sinne aus der Gefangenschaft in der Geschichte - und nicht mehr wie der Verfasser des Diario de a bordo fünfhundert Jahre zuvor aus der Gefangenschaft im Raum - entspringt. Von Amerika aus, so die düstere Einsicht und Aussicht Baudrillards, entsteht kein neues Bild Europas, Europa verschwindet vielmehr $(A, 32)^{34}$. Doch dürfte letztlich auch das Bild von einem Verschwinden jenes Projektors, der von Europa aus nach Westen gerichtet wurde, nichts anderes sein als ein amerikanischer Traum, von einem Europäer geträumt. $\mathrm{Ob}$ er zum Alptraum wird oder zu neuen Formen der Selbstvergewisserung am Anderen führt, können wir zuversichtlich dem neuen Millennium zur Klärung überlassen.

34 „En réalité, on ne prend pas ici, comme je l'espérais, de distance par rapport à l'Europe, on n'y gagne pas de point de vue plus étrange. Quand vous vous retournez, l'Europe a tout simplement disparu.« Anekdotenhalber sei erwähnt, dass der Verfasser im Besitz eines nordamerikanischen TShirts mit der Aufschrift »Columbus Quincentenary 1492-1992« ist, an dessen Rückenteil eine Erdkugel abgebildet wird, auf welcher man leicht den amerikanischen Kontinent, Afrika und Grönland, aber kein Europa mehr erkennen kann. Die drei überdimensioniert eingezeichneten Karavellen haben ihren Kurs nach Westen aufgenommen, wo überdies gerade die Sonne aufgeht, während über dem verschwundenen Europa nur noch eine magere Mondsichel und die Sterne leuchten. 


\section{Bibliographie}

Literarische Werke und andere Quellen

Baudrillard, Jean (1986): Amérique, Paris: Grasset.

Butor, Michel (1991): Mobile. Étude pour une représentation des États-Unis, Paris: Gallimard.

Colón, Cristóbal (1985): Diario de a bordo, hrsg. v. Luis Arranz, Madrid: Historia 16.

Cortés, Hernán (1985): Cartas de relación, hrsg. v. Mario Hernández, Madrid: Historia 16.

Ganivet, Ángel (1961): Ideárium español, in: id.: Obras Completas, hrsg. v. Melchor Fernández Almagro, Madrid: Aguilar, Bd. 1.

Humboldt, Alexander von (1836): Kritische Untersuchungen über die historische Entwickelung der geographischen Kenntnisse von der Neuen Welt und die Fortschritte der nautischen Astronomie in dem 15ten und 16ten Jahrhundert, 3 Bde., aus dem Französischen übersetzt von Dr. Jul. Ludw. Ideler, Privatdocent an der Berliner Universität, Berlin: Nicolaische Buchhandlung.

Humboldt, Alexander von (1970): Relation historique du voyage aux régions équinoxiales du Nouveau Continent fait en 1799, 1800, 1801, 1802, 1803, et 1804 par Al. de Humboldt et A. Bonpland, rédigé par Alexandre de Humboldt, 3 Bde., Neudruck des 1814-1825 in Paris erschienenen vollständigen Originals, besorgt, eingeleitet und um ein Register vermehrt von Hanno Beck, Stuttgart: Brockhaus.

Humboldt, Alexander von (1808): Über Steppen und Wüsten, in: id.: Ansichten der Natur mit wissenschaftlichen Erläuterungen, Tübingen: Cottasche Buchhandlung, Bd. 1 .

Humboldt, Alexander von (1986): Ansichten der Natur mit wissenschaftlichen Erläuterungen, Wiederabdruck der dritten, von Humboldt 1849 mit einem neuen Vorwort versehenen Ausgabe, Nördlingen: Greno.

Le Clézio, Jean-Marie Gustave (1988): Le rêve mexicain ou la pensée interrompue, Paris: Gallimard.

Núñez Cabeza de Vaca, Álvar (1984): Naufragios y Comentarios, hrsg. v. Roberto Ferrando, Madrid: Historia 16.

Masson de Morvilliers, Nicolas (1783): »Espagne«, in: Encyclopédie méthodique. Géographie, Paris / Lüttich: Panckoucke-Plomteux, Bd. 1, pp. 554-568.

Raynal, Guillaume-Thomas (1781): Histoire philosophique et politique des établissemens et du commerce des Européens dans les deux Indes, 10 Bde., Genf: Jean-Léonard Pellet.

Unamuno, Miguel de ( 1968 [1942]): »Del sentimiento trágico de la vida«, in: id.: Ensayos, 2 Bde., hrsg. v. Bernardo G. de Cándamo, Madrid: Aguilar, Bd. 2, pp. 727 1022 .

\section{Forschungsliteratur}

Barthes, Roland (1957): Mythologies, Paris: Seuil.

Barthes, Roland (1964): »Littérature et discontinu«, in: id.: Essais critiques, Paris: Seuil, pp. 175-187.

Eco, Umberto (1976): Opera aperta: forma e indeterminazione nelle poetiche contemporanee, Mailand: Bompiani. 
Ette, Ottmar (1988): »Papageien, Schriftsteller und die Suche nach der Identität. Auf den Spuren eines Vogels von Alexander von Humboldt bis in die Gegenwart«, in: Curiosités caraïbes. Festschrift für Ulrich Fleischmann, Berlin: Selbstverlag, pp. $35-40$.

Ette, Ottmar (1992): »Entdecker über Entdecker: Alexander von Humboldt, Cristóbal Colón und die Wiederentdeckung Amerikas«, in: Heydenreich, Titus (Hrsg.): Columbus zwischen zwei Welten. Historische und literarische Wertungen aus fünf Jahrhunderten, 2 Bde., Frankfurt am Main: Vervuert, Bd. 1, pp. 401-439.

Ette, Ottmar (1997): ') Est-ce que l'on sait où l'on va?< Dimensionen, Orte und Bewegungsmuster des Reiseberichts«, in: Bernecker, Walther L. / Krömer, Gertrut (Hrsg.): Die Wiederentdeckung Lateinamerikas. Die Erfahrung des Subkontinents in Reiseberichten des 19. Jahrhunderts, Frankfurt am Main: Vervuert, pp. 29-78.

Ferrando, Roberto (1984): »Introducción«, in: Núñez Cabeza de Vaca, Álvar: Naufragios y Comentarios, hrsg. v. Roberto Ferrando, Madrid: Historia 16, pp. 16-25.

Gewecke, Frauke (1986): Wie die neue Welt in die alte kam, Stuttgart: Klett-Cotta.

Jurt, Joseph (1982): »Entstehung und Entwicklung der LATEINamerika-Idee «, Lendemains 27 , pp. 17-26.

Phelan, John Leddy (1968): »Pan-Latinism, French Intervention in Mexico (1861-1867) and the Genesis of the Idea of Latin America«, in: Conciencia y autenticidad históricas. Escritos en homenaje a Edmundo O'Gorman, hrsg. v. Juan A. Ortega y Medina, Mexiko: UNAM, pp. 279-298.

Rodríguez Monegal, Emir (1982): „Die Neue Welt. Ein Dialog zwischen den Kulturen«, in: id.: Die Neue Welt. Chroniken Lateinamerikas von Kolumbus bis zu den Unabhängigkeitskriegen, Frankfurt am Main: Suhrkamp, pp. 7-65.

Rojas Mix, Miguel (1986): »Bilbao y el hallazgo de América latina: unión continental, socialista y libertaria«, Caravelle 46, pp. 35-47.

Todorov, Tzvetan (1985 [1982]): Die Eroberung Amerikas. Das Problem des Anderen, aus dem Französischen von Wilfried Böhringer, Frankfurt am Main: Suhrkamp. 